\title{
Isolation of Sesquiterpene Synthase Homolog from Panax ginseng C.A. Meyer
}

\author{
Altanzul Khorolragchaa, Shohana Parvin, Ju-Sun Shim, Yu-Jin Kim, Ok Ran Lee, \\ Jun-Gyo In, Yeon-Ju Kim, Se-Young Kim and Deok-Chun Yang* \\ Korean Ginseng Center and Ginseng Genetic Resource Bank, Kyung Hee University, \\ Seocheon, Giheung-gu Yongin-si, Gyeonggi-do 449-701, South Korea \\ (Received December 9, 2009; Revised February 5, 2010; Accepted February 8, 2010)
}

\begin{abstract}
Sesquiterpenes are found naturally in plants and insects as defensive agents or pheromones. They are produced in the cytosolic acetate/mevalonate pathway for isoprenoid biosynthesis. The inducible sesquiterpene synthases (STS), which are responsible for the transformation of the precursor farnesyl diphosphate, appear to generate very few olefinic products that are converted to biologically active metabolites. In this study, we isolated the STS gene from Panax ginseng C.A. Meyer, designated PgSTS, and investigated the correlation between its expression and various abiotic stresses using real-time PCR. PgSTS cDNA was observed to be 1,883 nucleotides long with an open reading frame of $1,707 \mathrm{bp}$, encoding a protein of 568 amino acids. The molecular mass of the mature protein was determined to be $65.5 \mathrm{kDa}$, with a predicted isoelectric point of 5.98. A GenBank BlastX search revealed the deduced amino acid sequence of PgSTS to be homologous to STS from other plants, with the highest similarity to an STS from Lycopersicon hirsutum (55\% identity, $51 \%$ similarity). Real-time PCR analysis showed that different abiotic stresses triggered significant induction of PgSTS expression at different time points.
\end{abstract}

Key words : Panax ginseng, sesquiterpene synthase (STS), abiotic stress, real-time PCR

\section{INTRODUCTION}

Sesquiterpenes, the $\mathrm{C}_{15}$ member of the terpenoid family of natural products, play a variety of ecological roles in higher plants. Those are derived from the cytosolic acetate/mevalonate pathway for isoprenoid biosynthesis. The inducible sesquiterpene synthases responsible for the transformation of the precursor farnesyl diphosphate (FPP) appear to generate very few olefinic products which are converted to biologically active metabolites, such as todomatuic acid, an insect hormone mimic that can alter larval and pupal development [1]. More than 300 types of cyclic sesquiterpenes have been characterized to date and each is derived from the common acyclic precursor FPP in a reaction catalyzed by a sesquiterpene synthase. Some of sesquiterpenes compounds exhibit antifeedant and antifungal activities [2]. In addition, many sesquiterpenes are volatile compounds that are commonly emitted from flowers and leaves of the plant that function as volatile cues to attract pollinators or parasitic and predatory insects [3]. Interestingly, volatile sesquiterpenes have also

\footnotetext{
* Corresponding author. E-mail: dcyang@khu.ac.kr
} Phone: +82-31-201-2100, Fax: +82-31-202-2687 been found to be synthesized and accumulated in rhizomes and roots or released from these below ground tissues [4]. Sesquiterpene synthases frequently appear to be rate-determining regulatory enzymes for the pathways in which they participate [5].

In the biosynthetic pathway of terpenes, various terpene synthases catalyze cyclization reactions, converting a few allylic diphosphates into a surprising array of cyclic products [6]. Gene cloning of several sesquiterpene synthases from different plant sources has been reported such as 5-epiaristolochene synthase from Nicotiana tabacum [7] and Capsicum аппиит [8], vetispiradiene synthase from Hyoscyamus muticus [9] and Solanum tuberosum [10], (1)-dcadinene synthase from Gossypium arboreum [6], and germacrene C synthase from Lycopersicon esculentum cv. VFNT Cherry tomato [11]. These plant terpene synthases exhibit a significant degree of similarity at the amino acid level [12].

Ginseng (Panax ginseng C. A. Meyer), a perennial herb from the Araliaceae family, is one of the most commonly utilized medicinal plants. The roots of ginseng plant harbor a host of pharmaceutical components including ginsenosides (saponins), polyacetylenes, polyphenolic compounds, and acidic polysaccharides [13]. In this study, we report 
on the isolation and characterization cDNA for the sesquiterpene synthase (STS) gene from $P$. ginseng and investigate the correlation between the PgSTS expression and different abiotic stresses by real-time PCR.

\section{MATERIALS AND METHODS}

\section{Plant materials}

Panax ginseng hairy roots were collected from Korean Ginseng Center and Ginseng Genetic Resource Bank, Kyung Hee University and cultured in hormone free liquid Murashige \& Skoog medium [14]. The roots were maintained by regular subculture in every 4 weeks. Abiotic stress treatment was carried out with one month subcultured roots.

\section{RNA purification and construction of a cDNA library}

Total RNA was isolated from a 4-year-old ginseng root by using the aqueous phenol extraction method [15]. Poly $(\mathrm{A})^{+}$RNA was isolated by oligo (dT) cellulose column using the Poly (A) Quick mRNA isolation kit (Stratagene, US). A commercial cDNA synthesis kit was used to construct library according to the manufacturer's instruction manual (Clontech, US). Size-selected cDNA was ligated into $\lambda$ TriplEx 2 vector and was packaged in vitro using Gigapack III Gold Packaging Extract kits (Stratagene, US).

\section{Nucleotide sequencing and sequence analysis}

The pTriplEx phagemids were excised from the $\lambda$ pTriplEx 2 and used as templates for sequence analysis. The 5' ends of cDNA inserts were sequenced by an automatic DNA sequencer (ABI prism 3700 DNA sequencer, Perkin-Elmer, USA). Homologous sequences of STS EST are searched against the GenBank databases using a BLASTX algorithm. A pTriplEx phagemid for STS cDNA was excised from the $\lambda$ pTriplEx 2 and used as templates for sequence analysis. Nucleotide and amino acid sequence analyses were performed using DNASIS program (Hitachi, Japan). The PgSTS gene was analyzed using softwares BioEdit, ClustalX, Mega3 and the other databases listed bellow; NCBI (http://www.ncbi.nlm.nih.gov/BLAST), ProtParam (http://us.expasy.org/tools/protparam.html), HMMTOP (http://www.enzim.hu/hmmtop), SOPMA (http:// npsa-pbil.ibcp.fr/cgi-bin/npsa_automat.pl?page=/NPSA/ npsa_server.html) and Prosite (http://www.expasy.ch/ prosite/) [16-18].

\section{Stress treatment}

To investigate the response of $P g S T S$ gene to various stresses, one month subcultured hairy roots were used. For chemical stress treatments, hairy roots were placed in MS [14] media containing indicated concentrations of chemicals; $100 \mathrm{mM}$ abscisic acid (ABA), $10 \mathrm{mM}$ salicylic acid (SA), and $0.2 \mathrm{mM}$ jasmonic acid (JA). Chilling stress was applied by exposing the hairy roots to a temperature of $4^{\circ} \mathrm{C}$. Stress treated plantlet samples were collected after $1,2,4,8,12,24$ and 48 hrs posttreatment. Control plants held in a growth room at $25^{\circ} \mathrm{C}$ under a $16 \mathrm{hrs}$ photoperiod. The stressed plant materials from all completed treatments were immediately frozen in liquid nitrogen and stored at $-70^{\circ} \mathrm{C}$ until required.

\section{Real-time PCR analysis}

Total RNA was extracted from stress treated hairy roots of $P$. ginseng using RNeasy mini kit (Qiagen, Valencia, CA, USA). For RT-PCR, $2 \mu \mathrm{g}$ of total RNA was used as a template for reverse transcription. Oligo $(\mathrm{dT})_{15}$ primer $(0.2 \mathrm{mM})$ (INTRON Biotechnology, Inc., South Korea) was added and the mixture was heated for $5 \mathrm{~min}$ at $75^{\circ} \mathrm{C}$. Then reaction mixture was incubated with AMV Reverse Transcriptase (10 U/ $\mu$ l) (INTRON Biotechology, Inc., South Korea) for $60 \mathrm{~min}$ at $42^{\circ} \mathrm{C}$. The reaction was inactivated by heating the mixture at $94^{\circ} \mathrm{C}$ for $5 \mathrm{~min}$. Realtime quantitative PCR was performed using $3 \mu \mathrm{l}$ of cDNA in a $10 \mu$ reaction volume using SYBR ${ }^{\circledR}$ Green Sensimix Plus Master Mix (Quantace, Watford, England). Specific primers for PgSTS, (forward) 5'- CTG GCC CGA AGA TTA ATG ACA AA -3' and (reverse) 5'- GAT GTC TAT ACT GAA ATG GAG GAA GAA ATG -3' were used. As a control, the primers specific to $P$. ginseng actin gene were used (forward, 5'- CGT GAT CTT ACA GAT AGC TTG ATG-3' and reverse, 5'- AGA GAA GCT AAG ATT GAT CCT CC-3'). The thermal cycler conditions recommended by the manufacturer were used as follow: $10 \mathrm{~min}$ at $95^{\circ} \mathrm{C}$, followed 40 cycles of $95^{\circ} \mathrm{C}$ for $10 \mathrm{~s}, 60^{\circ} \mathrm{C}$ for 10 $\mathrm{s}$, and $72^{\circ} \mathrm{C} 20 \mathrm{~s}$. The fluorescent product was detected at the last step of each cycle. Amplification, detection, and data analysis were carried out with a Rotor-Gene ${ }^{\mathrm{TM}} 6000$ real-time rotary analyzer (Corbett Life Science, Sydney, Australia). Threshold cycle $(\mathrm{Ct})$ represents the number of cycles at which the fluorescence intensity was significantly higher than the background fluorescence at the initial exponential phase of PCR amplification. To determine the relative fold differences in template abundance for each sample the $\mathrm{Ct}$ value for PgSTS was normalized to the $\mathrm{Ct}$ value for $\beta$-actin and calculated relative to a calibrator using the formula $2^{-\Delta \Delta \mathrm{Ct}}$. All experiments replicated 3 times. 


\section{RESULTS AND DISCUSSION}

\section{Isolation and characterization of a cDNA encoding PgSTS gene}

As part of a genomic project to identify genes of the medicinal plant $P$. ginseng, a cDNA library consisting about 20,000 cDNAs were previously constructed. A cDNA encoding Sesquiterpene Synthase (STS), designated PgSTS, was isolated and sequenced. As shown in Fig. 1, PgSTS is $1,883 \mathrm{bp}$ in length, and it has an open reading frame (ORF) of $1,707 \mathrm{bp}$ encodes a precursor protein of 568 amino acids. The ORF of PgSTS starts at nucleotide position 39 and ends at position 1,745. The calculated molecular mass of the matured protein is approximately 65.5 $\mathrm{kDa}$ with a predicted isoelectric point of 5.98. In the deduced amino acid sequence of PgSTS protein, the total number of negatively charged residues (Asp and Glu) was 80 while the total number of positively charged residues (Arg and Lys) was 65 (ProtParam).

\section{Homology and secondary structure analysis of pro- tein PgSTS}

A GenBank Blast search revealed that PgSTS has the highest sequence homology to Lycopersicon hirsutum STS (AAG41892) with identity 55\% and similarity 51\%. Sequence analysis of BLASTX program in NCBI database also showed high and similar identities with other species such as Lycopersicon esculentum (AAG41890) (identity $53 \%$ \& similarity 51\%), Santalum album (ACF24768) (identity 53\% \& similarity 50\%) and Fabiana imbricata (AAX40666) (identity 51\% \& similarity 48\%) (Fig. 2).

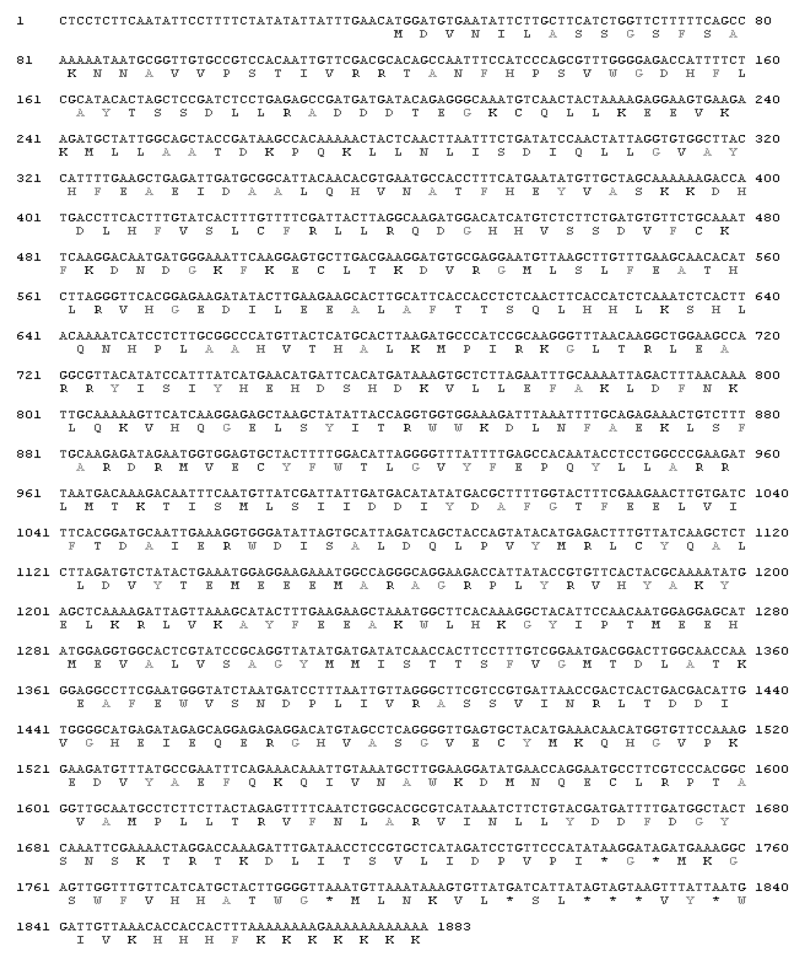

Fig. 1. Nucleotide sequence and deduced amino acid sequence of a PgSTS cDNA isolated from $P$. ginseng. Numbers on the left indicate nucleotide positions. The deduced amino acid sequence is shown below the nucleotide sequence, using the single letter code.

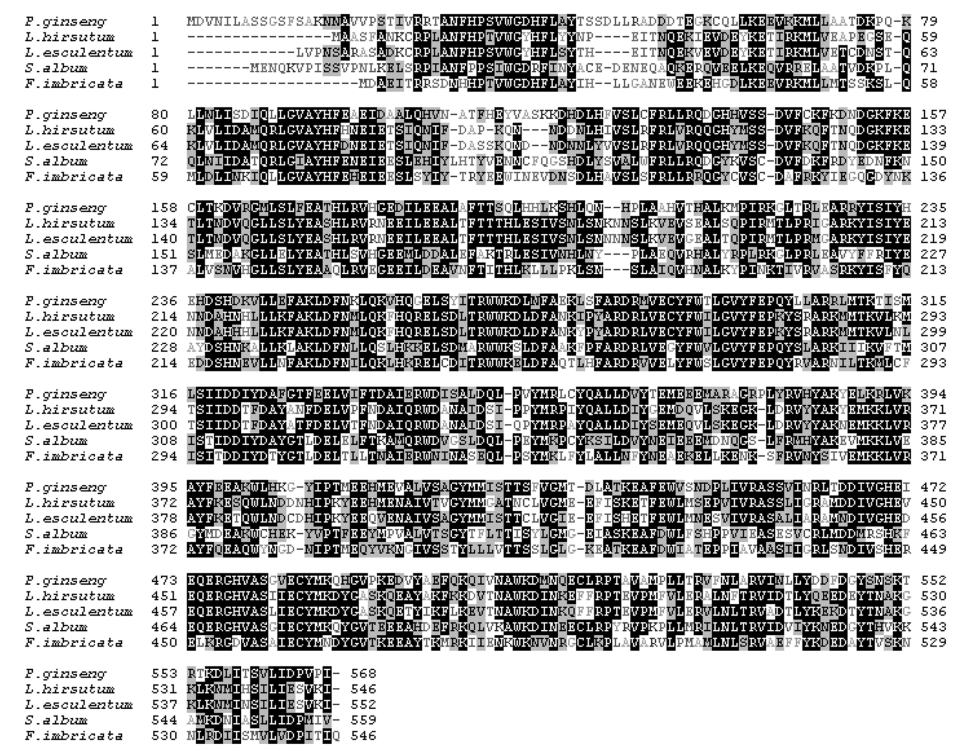

Fig. 2. Multiple amino acid sequence alignment of PgSTS with other closely related STS enzymes. The putative enzyme encoded by PgSTS shares a high degree of homology with enzymes from Lycopersicon hirsutum (AAG41892), Lycopersicon esculentum (AAG41890), Santalum album (ACF24768), and Fabiana imbricata (AAX40666). 
Secondary structure analysis and molecular modeling for PgSTS were performed by SOPMA. The secondary structure analysis revealed that PgSTS consists of $388 \alpha-$ helices, $17 \beta$-turns jointed by 27 extended strands, and 136 random coils. This is highly similar to the secondary structure of STSs of Lycopersicon hirsutum which contains $388 \alpha$-helices, $18 \beta$-turns jointed by 22 extended strands and 118 random coils; to Lycopersicon esculentum STSs which contains $369 \alpha$-helices, $19 \beta$-turns jointed by 32 extended strands and 132 random coils; to Santalum album STSs which contains $372 \alpha$-helices, $18 \beta$-turns jointed by 25 extended strands, and 144 random coils; to Fabiana imbricata STSs which contains $392 \alpha$-helices, $17 \beta$-turns jointed by 22 extended strands, and 115 random coils (Fig. 3).

ClustalX and the MEGA 3 Program were used for the construction of phylogenetic tree based on STSs amino acid sequences. PgSTS shares the highest homology with the STSs from tomatoes (L. hirsutum and L. esculentum) (Fig. 4).

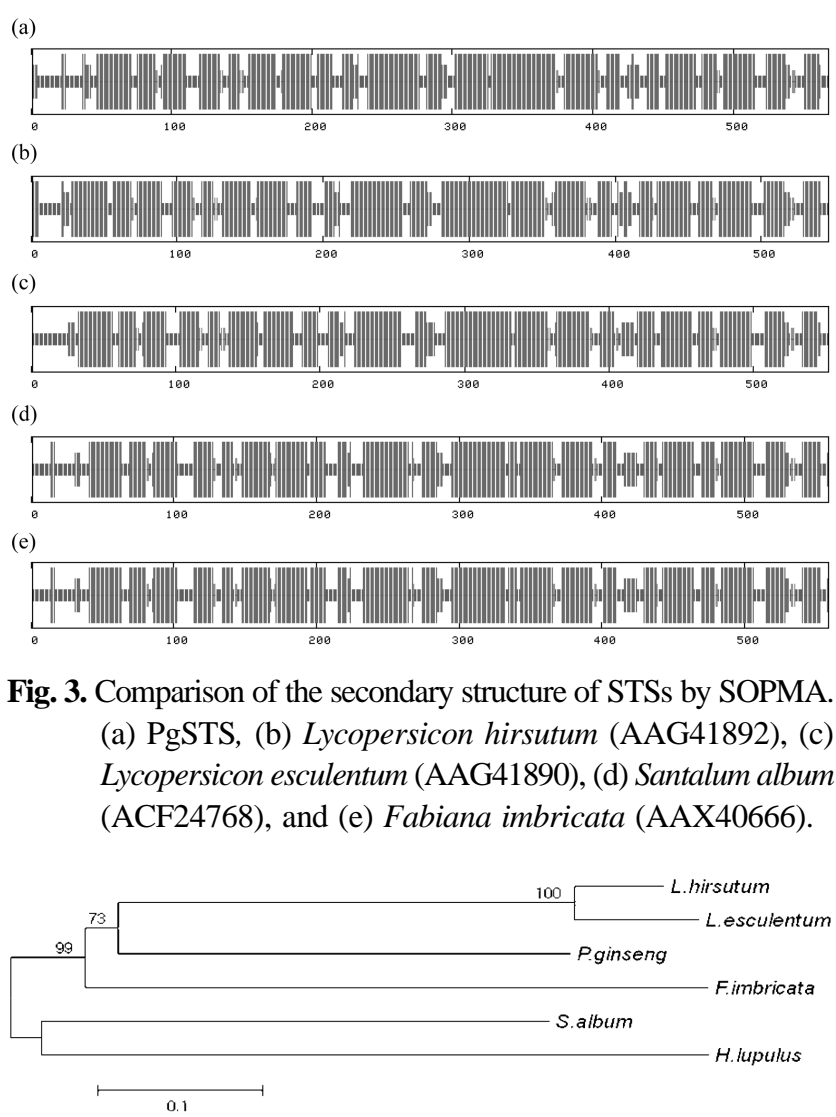

Fig. 4. A phylogenetic tree of PgSTS and other related STSs. The tree was constructed using ClustalX and the neighborjoining methods. The bar represents 0.1 substitutions per amino acid position.

\section{Differential expressions of the PgSTS under various abiotic stresses}

The expression patterns of the PgSTS under various abiotic stresses, such as stress-related chemicals including SA $(10 \mathrm{mM})$, ABA $(100 \mathrm{mM}), \mathrm{JA}(0.2 \mathrm{mM})$ and chilling $\left(4^{\circ} \mathrm{C}\right)$ were investigated by real-time PCR.

SA is a universal inducer of plant defensive metabolite production, it induces gene expression related to biosynthesis of some classes of secondary metabolites in plants [19]. Under SA treatment, the PgSTS expression increased till maximum 8.76 fold at $4 \mathrm{hr}$ post treatments and then the expression was gradually decreased until at $48 \mathrm{hrs}$ (Fig. 5a). Faldt et al. [20] reported that SA treatment did not induce any expression of terpene synthase in Arabidopsis thaliana. ABA, defined as a stress hormone, plays a central role in responses to biotic and abiotic stresses [21]. It was reported that $\mathrm{ABA}$ could stimulate the accumulation of some secondary metabolites [22, 23]. Under ABA treatment, the PgSTS expression level was increased to 3.56 fold at 2 $\mathrm{hr}$ and rapidly decreased to minimum value at $4 \mathrm{hr}$, and then the expression increased gradually to top point 4.56 fold at $48 \mathrm{hr}$ post treatments (Fig. 5b). Jasmonates have been reported to be elicitor signal transducers for production of plant secondary metabolites [24]. They induce accumulation of compounds belonging to different structural classes, including phenolics, terpenoids, alkaloids and others. For JA stress, the expression of PgSTS was increased $1 \mathrm{hr}$ with 1.47 fold and then gradually decreased (Fig. 5c). RT-PCR analysis showed the expression of AtTPSO3 at $16 \mathrm{hr}$ post treatments was higher than $2 \mathrm{hr}$ post treatments under JA treatment [20]. In chilling treatment, PgSTS transcript level was increased 2.95 fold at 1 $\mathrm{hr}$, and then decreased 2.20 fold at $2 \mathrm{hrs}$. The expression reached highest point at $4 \mathrm{hr}$ with 3.57 fold and then sustained as normal (Fig. 5d). Similar result showed in soybean, the gene expression of L-asparaginase was highly expressed after $6 \mathrm{hr}$ post treatments at low temperature [25].

In conclusion, we isolated STS gene from ginseng hairy root and characterized its expression in response to various stresses. PgSTS strongly induced by SA, ABA and chilling stresses. Therefore the expression level of $P g S T S$ revealed that STS gene may play a crucial role in protection of ginseng plant under environmental abiotic stresses.

\section{ACKNOWLEDGMENTS}

This study was supported by KGCMVP (Korean Ginseng Center for Most Valuable Products \& Ginseng) for 
(a)

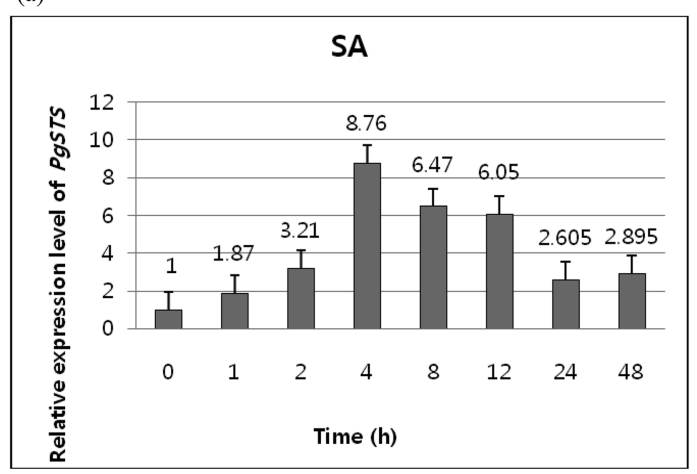

(b)

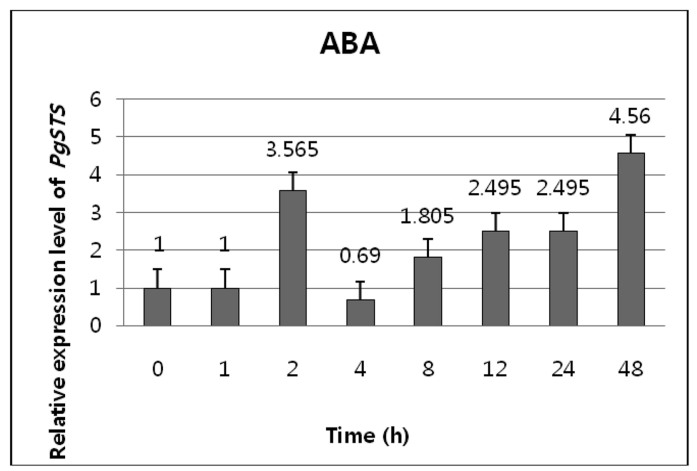

(c)

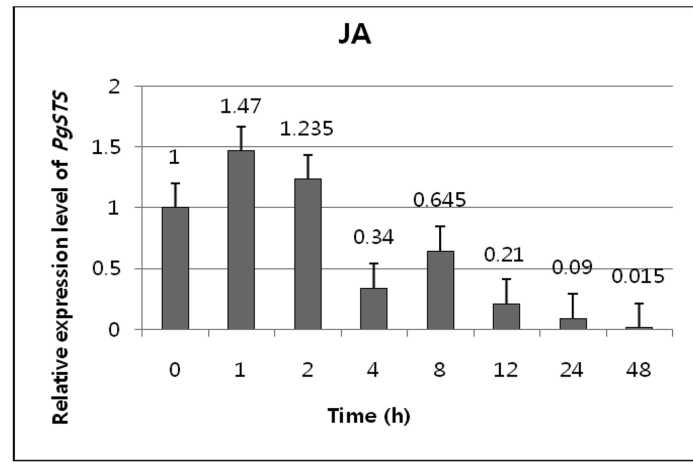

(d)

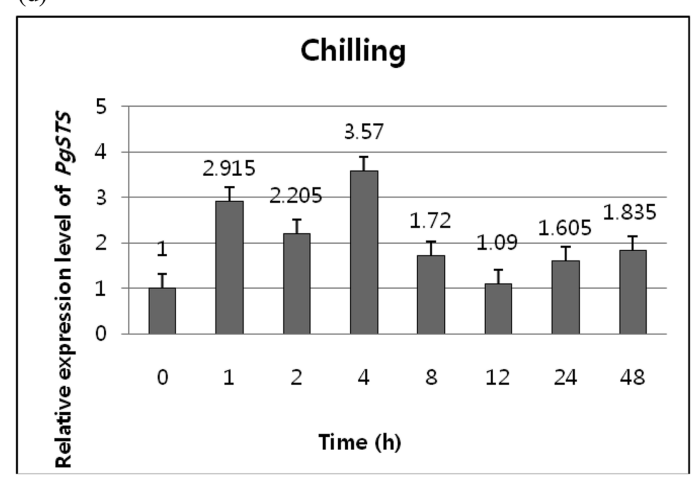

Fig. 5. Relative mRNA expression levels of PgSTS at various time points (h post-treatment) after stress. (a) SA (salicylic acid), $10 \mathrm{mM}$ treatment; (b) ABA (abscisic acid), $100 \mathrm{mM}$; (c) JA (jasmonic acid), $0.2 \mathrm{mM}$; and (d) chilling, $4^{\circ} \mathrm{C}$. The error bars represent the standard error of means of three independent replicates.

Technology Development Program of Agriculture and Forestry, Ministry for Food, Agriculture, Forestry and Fisheries, Republic of Korea.

\section{REFERENCES}

1. Yu F, Harada H, Yamasaki K, Okamoto S, Hirase S, Tanaka Y, Misawa N, Utsumi, R. Isolation and functional characterization of a $\beta$-eudesmol synthase, a new sesquiterpene synthase from Zingiber zerumbet Smith. FEBS Letters 582: 565-572 (2008)

2. Pechous SW, Whitaker BD. Cloning and functional expression of an (E,E)- $\alpha$-farnesene synthase cDNA from peel tissue of apple fruit. Planta 219: 84-94 (2004)

3. Cheng AX, Xiang CY, Li JX, Yang CQ, Hu WL, Wang LJ, Lou YG, Chen XY. The rice (E)- $\beta$-caryophyllene synthase (OsTPS3) accounts for the major inducible volatile sesquiterpenes. Phytochem. 68: 1632-1641 (2007)

4. Arimura GI, Garms S, Maffei M, Bossi S, Schulze B, Leither M, Mithofer A, Boland W. Herbivore-induced terpenoid emission in Medicago truncatula: concerted action of jasmonate, ethylene and calcium signaling. Planta 227: 453-464 (2008)
5. Picaud S, Olofsson L, Brodelius M, Brodelius PE. Expression, purification, and characterization of recombinant amorpha-4,11-diene synthase from Artemisia annua L. Arch Biochem Biophys. 436: 215-226 (2005)

6. Chen XY, Chen Y, Heinstein P, Davisson VJ. Cloning, expression, and characterization of $(+)-\delta$-cadinene synthase: a catalyst for cotton phytoalexin biosynthesis. Arch Biochem Biophys. 324: 255-266 (1995)

7. Facchini PJ, Chappell J. Gene family for an elicitor-induced sesquiterpene cyclase in tobacco. Proc Natl Acad Sci. 89: 11088-11092 (1992)

8. Back K, He S, Kim KU, Shin DH. Cloning and bacterial expression of sesquiterpene cyclase, a key branch point enzyme for the synthesis of sesquiterpenoid phytoalexin capsidiol in UV-challenged leaves of Capsicum annuum. Plant Cell Physiol. 39: 899-904 (1998)

9. Back K, Chappell J. Cloning and bacterial expression of a sesquiterpene cyclase from Hyoscyamus muticus and its molecular comparison to related terpene cyclases. J Biol Chem. 270: 7375-7381 (1995)

10. Yoshioka H, Yamada N, Doke N. cDNA cloning of sesquiterpene cyclase and squalene synthase, and expression of the genes in potato tuber infected with Phytophthora infestans. 
Plant Cell Physiol. 40: 993-998 (1999)

11. Colby SM, Crock J, Dowdle-Rizzo B, Lemaux PG, Croteau R. Germacrene C synthase from Lycopersicon esculentum cv. VFNT Cherry tomato: cDNA isolation, characterization, and bacterial expression of the multiple product sesquiterpene cyclase. Proc Natl Acad Sci. 95: 2216-2221 (1998)

12. Chang YJ, Song SH, Park SH, Kim SU. Amorpha-4,11-diene synthase of Artemisia annua: cDNA isolation and bacterial expression of a terpene synthase involved in artemisinin biosynthesis. Arch Biochem Biophys. 383: 178-184 (2000)

13. Kim MK, Lee BS, In JG, Sun H, Yoon JH, Yang DC. Comparative analysis of expressed sequence tags (ESTs) of ginseng leaf. Plant Cell Rep. 25: 599-606 (2006)

14. Murashige T, Skoog F. A revised medium for rapid growth and bio assay with tobacco tissue cultures. Plant Physiol. 15: 473-499 (1962)

15. Morris PC, Kumar A, Bowles DJ, Cuming AC. Osmotic stress and abscisic acid induce expression of the wheat Em genes. Eur J Biochem. 190: 625-630 (1990)

16. Alschul SF, Madden TL, Schaffer AA, Zhang J, Zhang Z, Miller W, Lipman DJ. Gapped BLAST and PSI-BLAST: a new generation of protein database search programs. Nucleic Acids Res. 25: 3389-3402 (1997)

17. Thompson JD, Gibson TJ, Plewniak F, Jeanmougin F, Higgins DG. The CLUSTAL_X windows interface: flexible strategies for multiple sequence alignment aided by quality analysis tools. Nucleic Acids Res. 25: 4876-4882 (1997)

18. Kumar S, Tamura K, Nei M. MEGA3: Integrated software for molecular evolutionary genetics analysis and sequence alignment. Brief in Bioinform. 5: 150-163 (2004)
19. Schenk PM, Kazan K, Wilson I, Anderson JP, Richmond T, Somerville SC, Manners JM. Coordinated plant defense responses in Arabidopsis revealed by microarray analysis. PNAS 97: 11655-11660 (2000)

20. Faldt J, Arimura G, Gershenzon J, Takabayashi J, Bohlmann J. Functional identification of AtTPSO3 as (E)- $\beta$-ocimene synthase: a monoterpene synthase catalyzing jasmonate and wound induced volatile formation in Arabidopsis thaliana. Planta 216: 745-751 (2003)

21. Smet ID, Zhang H, Inz D, Beeckman T. A novel role for abscisic acid emerges from underground. Trends in Plant Sci. 11: 434-439 (2006)

22. Smith RJ, Hobson S, Ellis IR. The effect of abscisic acid on calcium-mediated regulation of heterocyst frequency and nitrogenase activity in Nostoc 6720. New Phytologist 105: 543-549 (1987)

23. Jing F, Zhang L, Li M, Tang Y, Wang Y, Wang Y, Wang Q, Pan Q, Wang G, Tang K. Abscisic acid (ABA) treatment increases artemisinin content in Artemisia annua by enhancing the expression of genes in artemisinin biosynthetic pathway. Biologia 64: 319-323 (2009)

24. Gundlach H, Muller MJ, Kutchan TM, Zenk MH. Jasmonic acid is a signal transducer in elicitor-induced plant cell cultures. PNAS 89: 2389-2393 (1992)

25. Cho CW, Lee HJ, Chung ES, Kim KM, Heo JE, Kim JI, Chung J, Ma Y, Lee DW, Kim DH, Chung YS, Lee JH. Molecular characterization of the soybean L-asparaginase gene induced by low temperature stress. Mol Cells 23: 280286 (2007) 